\title{
ASPECT: A spectra clustering tool for exploration of large spectral surveys ${ }^{\star}$
}

\author{
A. in der Au, H. Meusinger, P. F. Schalldach, and M. Newholm
}

\author{
Thüringer Landessternwarte Tautenburg, Sternwarte 5, 07778 Tautenburg, Germany \\ e-mail: aick@texture-editor.com
}

Received 6 July 2012 / Accepted 13 September 2012

\section{ABSTRACT}

\begin{abstract}
Context. Analysing the empirical output from large surveys is an important challenge in contemporary science. Difficulties arise, in particular, when the database is huge and the properties of the object types to be selected are poorly constrained a priori. Aims. We present the novel, semi-automated clustering tool ASPECT for analysing voluminous archives of spectra.

Methods. The heart of the program is a neural network in the form of a Kohonen self-organizing map. The resulting map is designed as an icon map suitable for the inspection by eye. The visual analysis is supported by the option to blend in individual object properties such as redshift, apparent magnitude, or signal-to-noise ratio. In addition, the package provides several tools for the selection of special spectral types, e.g. local difference maps which reflect the deviations of all spectra from one given input spectrum (real or artificial). Results. ASPECT is able to produce a two-dimensional topological map of a huge number of spectra. The software package enables the user to browse and navigate through a huge data pool and helps them to gain an insight into underlying relationships between the spectra and other physical properties and to get the big picture of the entire data set. We demonstrate the capability of ASPECT by clustering the entire data pool of $\sim 6 \times 10^{5}$ spectra from the Data Release 4 of the Sloan Digital Sky Survey (SDSS). To illustrate the results regarding quality and completeness we track objects from existing catalogues of quasars and carbon stars, respectively, and connect the SDSS spectra with morphological information from the GalaxyZoo project.
\end{abstract}

Key words. methods: data analysis - surveys - white dwarfs - stars: carbon - quasars: general

\section{Introduction}

Astronomy has become a data-intensive science. Cutting edge research is requiring in particular deep and/or wide surveys producing data of unprecedented quality and volume. The Sloan Digital Sky Survey, (SDSS; Abazajian et al. 2009), one of the most ambitious and influential astronomical surveys, obtained more than $10^{6}$ spectra of galaxies and quasars. With the growth of massive data-producing sky surveys such as e.g., the Large Synoptic Sky Survey (LSST Science Collaborations et al. 2009), astronomical research will become even more data-intensive in the near future. Berriman \& Groom (2011) predict a growth rate of 0.5 petabyte of electronically accessible astronomical data per year. For example, vast and deep surveys using multi-object wide-field spectrographs, mainly on large aperture telescopes, will be critical for attempts to constrain the nature of dark matter, dark energy, and the processes of large-scale structure formation (Peacock et al. 2006; Bell et al. 2009; Morales et al. 2012).

Analysing the observational output from a large survey is greatly hindered by the sheer size of the data volume. For example, it is desirable to visualise the output in a big picture that illustrates both the diversity of the object types, their differences and similarities, but also correlations with certain physical parameters at once. The selection of the objects of a given spectral type among hundreds of thousands or even millions of spectra provides another problem. In principle, this job can be done by using the output from an efficient automated spectroscopic pipeline (e.g., Stoughton et al. 2002). In the case of

* Code is only available at the CDS via anonymous ftp to cdsarc.u-strasbg.fr (130.79.128.5) or via

http://cdsarc.u-strasbg.fr/viz-bin/qcat?J/A+A/547/A115 particularly interesting, rare object types with poorly constrained spectral features, however, it is not a priori clear if one can trust the pipeline. For instance, Hall et al. (2002) had to inspect 120000 spectra visually to find out 23 broad absorption line quasars with various unusual properties.

We developed a new software tool that is able to organise large spectral data pools by means of similarity in a topological map. The tool reduces the effort for visual inspection, enables easier selection from vast amounts of spectral data, and provides a greater picture of the entire data set. The approach is based on similarity maps generated using self-organising maps (SOM) as developed by Kohonen (2001). The SOM technique is an artificial neural network algorithm that uses unsupervised learning in order to produce a two-dimensional mapping of higher order input data.

Neural networks have been extensively used in the field of astrophysics, primarily for different kinds of classification tasks. Odewahn et al. (1992) were the first who applied multilayer perceptrons with backpropagation for an image-based discrimination between stars and galaxies. Mähönen \& Hakala (1995) and Miller \& Coe (1996) pioneered the use of SOMs for the same purpose, and Andreon et al. (2000) continued with work in this field. Further, SOMs have been used for classification of light curves (Brett et al. 2004), gamma-ray bursts (Balastegui et al. 2001; Rajaniemi \& Mähönen 2002), stellar spectra (Xue et al. 2001), stellar populations (Hernandez-Pajares \& Floris 1994), and broad absorption line quasar spectra (Scaringi et al. 2009) using learning vector quantization, a supervised generalisation of SOMs. However, the application of this type of neural network is not only limited to classification tasks. For instance, Lesteven et al. (1996) applied SOMs to organise astronomical 
publications, Naim et al. (1997) visualised the distribution of galaxies, Way \& Klose (2012) and Geach (2012) estimated photometric redshifts, and Torniainen et al. (2008) analysed gigahertz-peaked spectrum (GPS) sources and high frequency peakers (HFP) using SOMs in order to find homogeneous groups among the sources. For a more complete survey of neural network applications in astronomy, we refer to Tagliaferri et al. (2003) and Ciaramella et al. (2005).

In most studies found in the literature, neural networks have been used for some sort of object type classification. Therefore, a given source sample - that consists either of the entire spectra or some associated physical properties - is divided into a training and a test data set. Then, a small network with a few hundred neurons is trained with the training data set and then, the error rate of the classifier is estimated with the second data set. Our approach goes beyond this technique since we use the network to generate a map that contains every single optical spectrum of the source data pool grouped by similarity.

To achieve this goal, our network has to consist of orders of magnitude more neurons as compared to networks that are used for classification tasks. According to our knowledge, common software packages, for instance SOM Toolbox for Matlab ${ }^{1}$, SOM_PAK ${ }^{2}$ or commercial ones such as Peltarion ${ }^{3}$ are not capable of handling such large networks, so we decided to develop our own software.

This paper presents the new software tool ASPECT (A SPEctra-Clustering Tool) for computing and evaluating of very large SOMs. The overall process consists of the following steps: 1 . Selection and preparation of the spectral data set; 2. preprocessing of the spectra; 3 . computing the SOM; 4. visualisation and exploration of the final map. The last step includes such options as blending selected parameters (e.g., coordinates, object type, redshift, redshift error, ...) over the map, selecting objects from user-defined regions of the map, identifying objects from an external catalogue, or searching for spectra of a special type defined by a template spectrum.

In the next section, we discuss the selection and preparation of our example spectral data set. Section 3 describes the used algorithms to generate a SOM for $\sim 10^{6}$ spectra and discusses some important implementation details and optimisations necessary in order to finish computations in a reasonable time frame. Then, in Sect. 4, we explain the strength of such a SOM and show some visualisations of physical properties attached to each spectrum. Further we demonstrate the application of our approach for searching rare spectral types using carbon stars from the catalogues of Koester \& Knist (2006) and Downes et al. (2004). Finally, in Sect. 5, we shortly discuss two example applications for our SOM: The search for unusual quasars, and then, by connecting the SOM with morphological data from the Galaxy Zoo project (Lintott et al. 2011a), we illustrate how the achieved results can be combined with external data sets from different scientific works.

\section{Database, selection and preparation of the spectral data set}

\subsection{Database: the Sloan Digital Sky Survey}

The Sloan Digital Sky Survey (SDSS; York et al. 2000) is currently one of the most influential surveys in modern astronomy,

\footnotetext{
wWW. cis . hut. fi/somtoolbox

2 www.cis.hut.fi/research/som_pak

wWw peltarion. com
}

especially in the extragalactic domain. The SDSS provides photometric and spectroscopic data for more than one quarter of the sky. The survey started in 1998 and has a spectroscopic coverage of 9274 square degrees. The Data Release 8 (Aihara et al. 2011 ) contains spectra of over $1.6 \times 10^{6}$ galaxies, quasars, and stars. Imaging and spectroscopic data were taken with the $2.5 \mathrm{~m}$ telescope at Apache Point Observatory, New Mexico. The telescope is equipped with two digital fiber-fed spectrographs that can observe 640 spectra at once. Photometric data, processed by automatic imaging pipelines (Lupton et al. 2001) was later used to select spectra of different object classes (quasars, galaxies, luminous red galaxies, stars and serendipitous objects). Observed spectra were further automatically processed by a spectroscopic pipeline which reduces, corrects, and calibrates the spectra. For each spectrum the pipeline determined its spectral type and measured redshift, emission, and absorption lines.

The completion of the original goals of the SDSS and the end of the phase known as SDSS-II is marked by the DR7 (Abazajian et al. 2009). We started our study on Kohonen mapping of the SDSS spectra at the time of the DR6 (Adelman-McCarthy \& the SDSS Collaboration 2008) which contains over 1.2 million spectra. The early attempts were aimed at a basic understanding of the SOMs rather than analysing the complete set of spectra from the latest SDSS data release. We thus used the smaller database from the DR4 (Adelman-McCarthy et al. 2006) with about $8 \times 10^{5}$ spectra. Later on, we used the $\sim 10^{5}$ quasar spectra from the DR7 for a special application of ASPECT to create a sizeable sample of unusual SDSS quasars (Meusinger et al. 2012; Sect. 5.1). The aim of the present study, namely demonstrating the power and the general properties of the SOMs for all types of objects from the SDSS spectroscopic survey, does not require to involve the complete database from the last data release. We decided again to use the database from the DR4 simply in order to reduce the size of the complete map as well as the corresponding computing time to a manageable size. The spectra itself were taken from the DR6, which operates on an improved spectroscopic pipeline over DR4. Creating the here presented DR4 map took over 100 days computing time on a single workstation ${ }^{4}$ whereas a runtime of nearly 3 years is estimated for the corresponding map from the DR7. This problem for SDSS DR8 or upcoming data releases could be overcome in two ways. Either by clustering multiple smaller maps in parallel, each map on a different workstation, or by distributing the computational workload for one large map onto multiple workstations so that computing times are reduced to a manageable length. Our current software prototype executes already several algorithms in parallel on a single multi-core or multiprocessor machine. However distributed computations among multiple computers are not yet supported.

The SDSS spectra cover the wavelength range from $3800 \AA$ to $9200 \AA$ with a resolution of $\sim 2000$ and a sampling of $\sim 2.4$ pixels per resolution element. Each spectrum is given as a FITS file and can be identified by the combination of its MJD, plate number, and fiber id. In addition to the observed spectrum, each FITS file contains a rich set of parameters and physical properties where we are interested in a small fraction only.

All spectra are stored in the SpecObjAll database table. In order to eliminate useless or undesired spectra, we only took those from the SpecObj database view. According to Gray et al. (2002) duplicate objects, plates for quality assurance, sky data or plates that are outside the official survey boundaries are removed in this view. During preprocessing we then had

4 Intel Core i7 920 at $2.67 \mathrm{GHz}$ with $12 \mathrm{~GB}$ RAM. 
to remove additional 21 objects where pixels contained either infinite numbers or NANs (not a number) in their spectrum. Our final sample includes 608793 spectra; these are $90 \%$ of the DR4 spectroscopy main survey.

\subsection{Preprocessing of spectral data}

The preprocessing was performed in 3 steps:

(1) We reduced the overall size of the data pool to a necessary minimum by writing only required data (spectrum, redshift, spectra classification, MJD, plate id, fiber id) into a single binary file. Other data items from the FITS file, for instance emission lines, continuum-subtracted spectrum, noise in spectrum, mask array, and header information were omitted.

(2) The spectra were rebinned to reduce the number of pixels by a factor of 8 and the overall file size from $182 \mathrm{~KB}$ to $2 \mathrm{~KB}$ per spectrum (117 GB to $1.1 \mathrm{~GB}$ total). This reduction was done by taking the average of two pixels $S_{j}=\left(Y_{2 j}+Y_{\min (2 j+1, n)}\right) / 2$ for $j=1$ to $n / 2$, where $S_{j}$ is the $j$ th pixel in the smoothed spectrum, $Y_{j}$ the $j$ th pixel in the original spectrum, and $n=3900$ the number of pixels. The smoothing was applied iteratively three times over each spectrum. For the applications discussed in this paper (search of unusual quasars and carbon stars), the full spectral resolution is not necessary because we are looking for unusual continua or broad absorption or emission features which are usually at least one order of magnitude broader than the spectral resolution element of the original SDSS spectra. Since the SOM algorithm has to project every single spectrum into a two-dimensional plane only the continuum and the most prominent features are considered and several trade-offs have to be made. Indeed the algorithm is very efficient at this task but it cannot consider every small spectral feature of every input spectrum. Therefore, the reduction of the spectral resolution caused by the rebinning does not significantly reduce the quality of the clustering results as initial tests have shown. On the other hand, some applications may require the full spectral resolution. One solution would be trading spectral coverage against spectral resolution. For instance Scaringi et al. (2009) use a small spectral window from $1401 \AA$ to $1700 \AA$ for the classification of BALQSOs.

(3) We normalised each spectrum by the total flux density, i.e. the flux density integrated over the whole spectrum. To remove gaps of bad pixels that are not marked as OK or emission line in the mask array, we used a similar technique as proposed by Xue et al. (2001). These gaps were linearly interpolated before the reduction process was done.

To mention in passing, we do not transform the spectra into their restframes. The main reason is that stars and high-redshift extragalactic objects usually share only a narrow restframe wavelength interval; there is no wavelength overlap at all for quasars with redshift $z \gtrsim 1.5$ and sources at $z \sim 0$. Further, the observed spectra are independent of wrong redshift determinations from the spectroscopic pipeline.

\section{Computation of the SOM}

In this section, we describe the generation of the SOM for about $6 \times 10^{5}$ spectra from the SDSS DR4, which is a big challenge due to its sheer size. The SOM is a very effective algorithm that transforms non-linear statistical relationships of the original

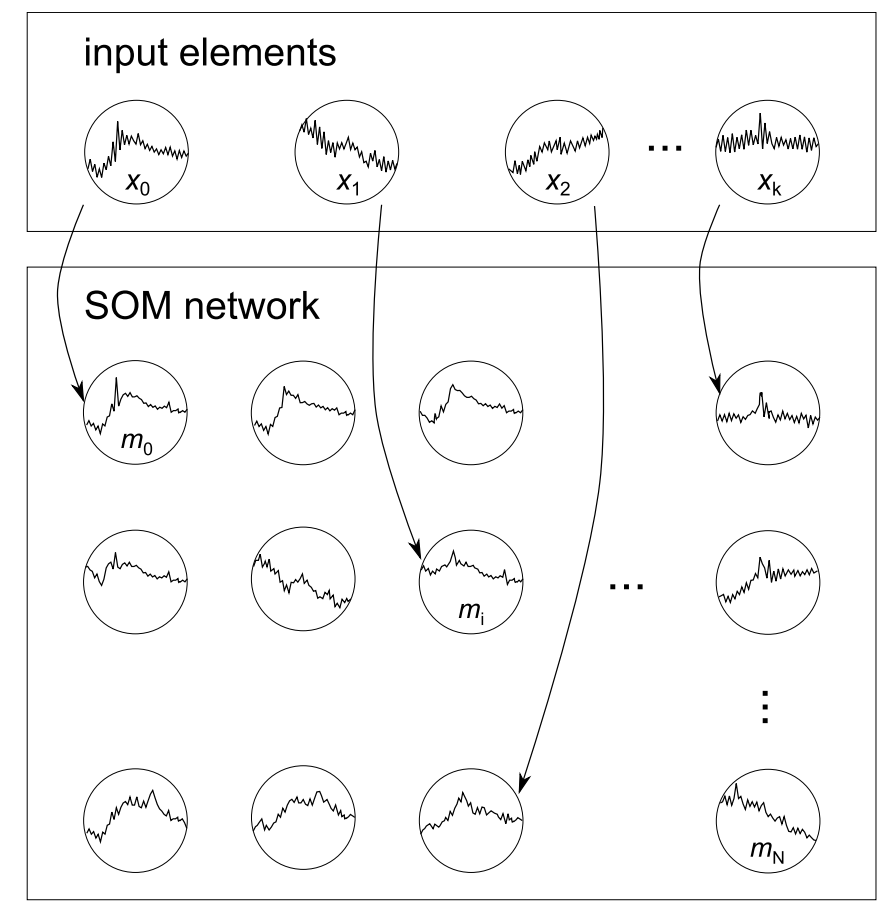

Fig. 1. SOM network layout: the two-dimensional array of neurons $\boldsymbol{m}_{\mathrm{i}}$.

high-dimensional input data (here: spectra) into simple geometric relationships in the resulting two-dimensional map, which consists of all input spectra ordered by their appearance.

As it is a basic property of SOMs that objects of the same "spectral type" tend to form conglomerates and clusters, we denote the whole process as "clustering". First, we will briefly describe the basic algorithm and its mathematical model; for a full discussion we refer to Kohonen $(1982,2001)$ from where the mathematical notation was adopted. Then, in the next section, we discuss in-depth all necessary implementation details and considerations taken into account.

\subsection{The SOM model for spectral clustering}

The set of input variables is defined as vectors $\boldsymbol{x}(j)=$ $\left[\xi_{1}(j), . ., \xi_{n}(j)\right]^{T} \in \mathfrak{R}^{n}$ where $n=488$ is the number of pixels in each reduced spectrum and $j$ denotes the index in the sequence of source spectra running from 0 to $k=608792$. The neural network then consists of $i \in\{1 . . N\}$ neurons, represented by weight vectors $\boldsymbol{m}_{i}(T)=\left[\mu_{i 1}(T), . ., \mu_{i n}(T)\right]^{T} \in \mathfrak{R}^{n}$, that are organised on a two-dimensional grid and $T=0,1,2, \ldots$ is the discrete time coordinate.

Typically, neurons are organised on a hexagonal lattice. However, we have chosen a rectangular lattice, since it allows easier and more compact visualisation of our resulting maps as simple rectangular images. Regarding boundary conditions a flat grid performs best, experiments with cylindrical and toroidal topologies reduced the quality of the clustering. Figure 1 shows the basic network layout with the two-dimensional array of neurons $\boldsymbol{m}_{\mathrm{i}}$. Each input element $\boldsymbol{x}(j)$ is associated with its best matching neuron at every discrete time step $T$. A fraction of neurons is empty (has no association with input elements) because $N>k$. A detailed discussion about the reasons is postponed to Sect. 3.2.2.

The process can be initialised by pure randomly chosen weight vectors but such an initialisation policy is not the fastest as stated by Kohonen (2001). We found that the number of 


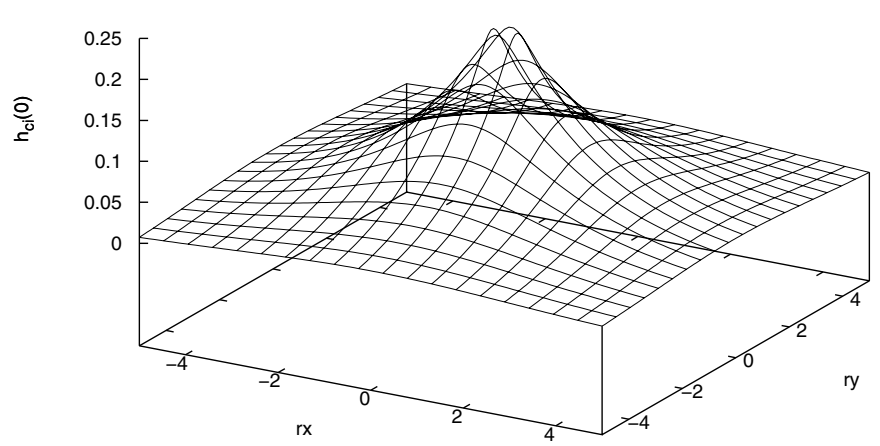

Fig. 2. The neighbourhood function $h_{\mathrm{ci}}$ at time $t=0$ as a function of the normalised radial distances to the BMU, $r_{x}$ and $r_{y}$ where the value 1.0 corresponds to the map size.

necessary training steps is substantially reduced by initialising each weight vector $\boldsymbol{m}_{i}(0)$ with a random input spectrum $\boldsymbol{x}(j)$.

The basic SOM algorithm is then based on two important processes that are responsible for the self-organising properties of the neural network: first choosing a winner neuron $\boldsymbol{m}_{c}$ among all $\boldsymbol{m}_{i}$ that has the best match to a given spectrum $\boldsymbol{x}$. Second, adaption of all neurons in the neighbourhood of $\boldsymbol{m}_{c}$ towards $\boldsymbol{x}$. For each learning step we present each $\boldsymbol{x}(j)$ in a random order to the network and compute the Euclidean distances $\left\|\boldsymbol{x}-\boldsymbol{m}_{i}\right\|$ to each neuron $\boldsymbol{m}_{i}$ as a measure of dissimilarity. Then, the best matching unit (BMU) is defined by the shortest Euclidean distance

$c=\underset{i}{\arg \min }\left\{\left\|\boldsymbol{x}-\boldsymbol{m}_{i}\right\|\right\}$.

To prevent collisions in the search for BMUs, where two or more different input spectra would share the same neuron, only such neurons $\boldsymbol{m}_{i}$ are considered that do not match with any of the previously presented input vectors. The iterated presentation of input vectors in random order over many learning steps ensures fairness among all inputs. In contrast with a constant sequence, some input vectors would receive higher priorities because they appear at the beginning of the sequence.

Then the BMU and all neurons in the neighbourhood are updated according to

$\boldsymbol{m}_{i}(T+1)=\boldsymbol{m}_{i}(T)+h_{\mathrm{ci}}(t)\left(\boldsymbol{x}-\boldsymbol{m}_{i}(T)\right)$,

with $t=T / T_{\max }$ and where the neighbourhood function

$h_{\mathrm{ci}}(t)=\alpha(t) \cdot \exp \left(-\frac{\left\|\boldsymbol{r}_{c}-\boldsymbol{r}_{i}\right\|}{2 \sigma^{2}(t)}\right)$

acts as a smoothing kernel over the network. With increasing number of learning steps, $h_{\mathrm{ci}}(t)$ approaches zero for convergence. Figure 2 shows the neighbourhood function for the first learning step. $\boldsymbol{r}_{c} \in \mathfrak{R}^{2}$ is the location vector of the BMU and $\boldsymbol{r}_{i} \in \mathfrak{R}^{2}$ the location vector of weight vector $\boldsymbol{m}_{i}$.

Compared to the frequently used Gaussian kernel, our kernel has broader wings and a sharper peak at its centre. We found from various trials that Eq. (3) yields better clustering results than its Gaussian counterpart. For one-dimensional networks, Erwin et al. (1992) have shown that convergence times are minimal for broad Gaussian neighbourhood functions. Employing a function that begins with a large width of the order of the largest dimension of the network allows rapid formation of an ordered

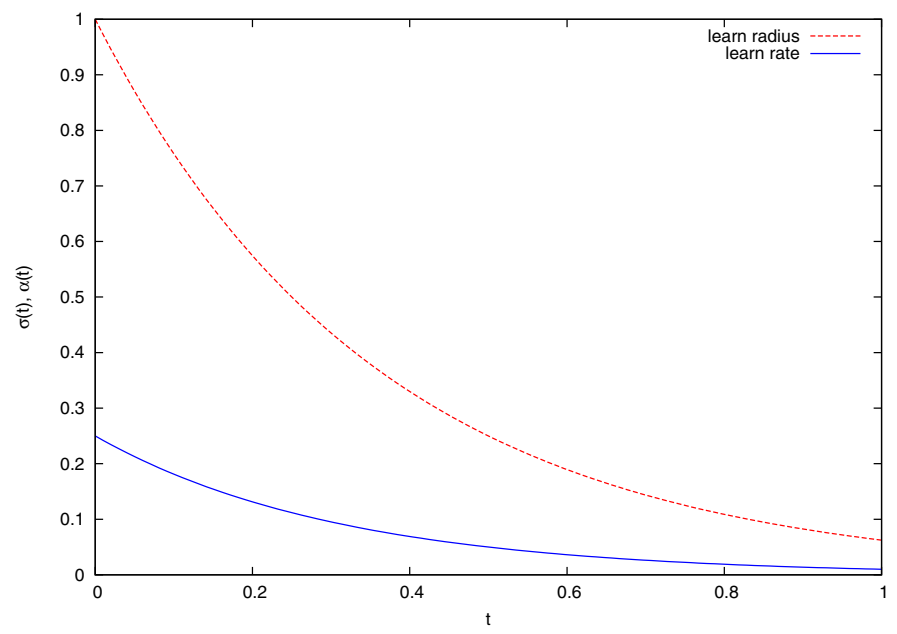

Fig. 3. Learn radius function $\sigma(t)$ and learn rate function $\alpha(t)$ with parameters $\sigma_{\text {begin }}=1.0 \sigma_{\text {end }}=0.0625, \alpha_{\text {begin }}=0.25, \alpha_{\text {end }}=0.01$.

map. This is a consequence of the absence of metastable stationary states 5 , which slow down the convergence progress by orders of magnitudes. After an ordered map is formed in the first learning steps the width of the kernel can be reduced to develop small-scale structures within the map.

The neighbourhood function is modified over time by the learn rate function

$\alpha(t)=\alpha_{\text {begin }}\left(\frac{\alpha_{\text {end }}}{\alpha_{\text {begin }}}\right)^{t}$

and the learn radius function

$\sigma(t)=\sigma_{\text {begin }}\left(\frac{\sigma_{\text {end }}}{\sigma_{\text {begin }}}\right)^{t}$.

Both functions are monotonically decreasing over the time $t=$ $0 \ldots 1$ altering the neighbourhood function in such a way that large-scale structures form in the early training phase while small-scale structures and finer details appear at later training steps. Figure 3 shows booth functions for the start and end parameters used for the clustering process.

The parameters on the right-hand side of Eqs. (4) and (5) are the learning parameters of our Kohonen network (with $\alpha_{\text {begin }} \geq$ $\alpha_{\text {end }}$ and $\sigma_{\text {begin }} \geq \sigma_{\text {end }}$ ). In Sect. 3.2, we describe a mechanism how those parameters can be chosen properly. In order to keep network parameters $\sigma_{\text {begin }}$ and $\sigma_{\text {end }}$ scale-invariant regarding the number of neurons within the network, the distance term in $h_{\mathrm{ci}}(t)$ should be normalised to the grid size. This can be useful when experimenting with different network sizes.

The crucial information of this process is the mapping of input spectra to BMUs within the rectangular organised network. After a certain number of learning steps, the ordering has taken place and source spectra get mapped to the same network location over and over again. Jumps to different areas in the map are rare. At this point we obtain the ordered map of input spectra as result (see Sect. 3.2.4).

\subsection{Implementation details}

Before the computation can start, we have to specify all network parameters listed in Table 1. Owing to the long computation time

5 States where the energy function of the weight vectors, i.e. their change rate, reaches a local minimum instead of a global one (Erwin et al. 1992). 
Table 1. Network parameters used for final clustering.

\begin{tabular}{lc}
\hline \hline Number of neurons $N$ & $859 \times 859$ \\
Number of learning steps $T_{\max }$ & 200 \\
Learn radius $\sigma_{\text {begin }}$ & 1.0 \\
Learn radius $\sigma_{\text {end }}$ & 0.0625 \\
Learning rate $\alpha_{\text {begin }}$ & 0.25 \\
Learning rate $\alpha_{\text {end }}$ & 0.01 \\
\hline
\end{tabular}

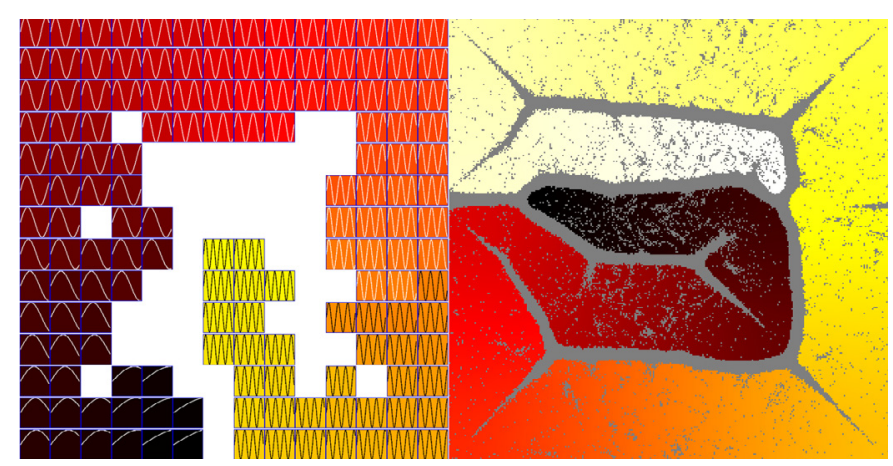

Fig. 4. Clustering of sinusoidal test spectra with $N=196$ and $k=150$ (left) and $N=96721$ (right), respectively.

of 108 days, it is not possible to tweak the network parameters and repeat the entire computation several times until a satisfying result in terms of accuracy and convergence is reached. Ideally, the clustering of the huge database should be done in one shot without successive recomputations.

\subsubsection{Deduction of network parameters}

Therefore we deduced all parameters by using a smaller set of artificial test "spectra" containing sinusoidal signals with increasing frequencies $f$ as input data. The limiting frequencies $f_{\min }$ and $f_{\max }$ were chosen arbitrary in a way so that oscillation is visible and no aliasing artefacts occur on weight vectors $\boldsymbol{m}_{i}$. This test setting permits to tweak all network parameters and shows clearly the goodness of a produced clustering. As a success criterion it is required that all test spectra settle finally in one coherent structure, sorted by their frequency.

The best results show a cluster that forms some sort of Hilbert style curve. The left part of Fig. 4 shows the final clustering result of a $14 \times 14$ map with 140 input elements. For validation purposes we repeated this test with the same parameter combination for greater sets of test spectra. The right panel of Fig. 4 shows the clustering behaviour of 80000 sinusoidal test spectra on a map with $311 \times 311$ cells. Empty cells are marked grey, frequencies are colour-mapped from black, red, yellow to white, where black denotes the lowest frequency.

Experience from many trials with smaller maps and real spectra have shown that good clustering results can be achieved with parameter combinations that performed well with the "sinusoidal" test setting and worse results are achieved with parameter combinations that performed poor in the above described test setting. However to our knowledge there exists no mathematical proof of the convergence properties of the SOM for the general case, i.e. $n$-dimensional input data on a twodimensional map. A proof for the one-dimensional case on an one-dimensional network with a step-neighbourhood function was given by Cottrell \& Fort (1987), Cottrell et al. (1994) review the theoretical aspects of the SOM.

\subsubsection{Considerations regarding the size of the network}

The number $N$ of neurons in the network must be at least equal to the number of source spectra in order to guarantee an injective mapping of source spectra. However initial tests showed that better results can be achieved if some cells are not occupied with source spectra. For such cells the neurons are not linked to source spectra. In the evolution of the neural network, such empty neurons lead to a better separation between distinct clusters because they tend to settle at the cluster boundaries. The same behaviour is observed for small groups and even for single outlier spectra. Another important factor is the decrease of probability for collisions of BMUs when two or more source spectra want to occupy the same neuron. Too many empty neurons, on the other hand, (1) scatter similar source spectra too much across the map so that no clear cluster boundaries may evolve; and (2) significantly increase the computing time. A factor of $N / k \approx 1.2$ produces a good trade-off where similar source spectra are not scattered too much but still have enough room to get into the right clusters.

\subsubsection{Optimisation techniques for faster computations}

We used two optimisation techniques in order to finish the computation in a reasonable time frame. The first technique speeds up the search phase from $O\left(N^{2}\right)$ up to $O(N)$ for the last learning step. For the first learning steps $(T<5)$ we conducted a full search which requires $\sim k N$ operations per learning step. Each operation requires the calculation of the Euclidean distance of a source spectrum - weight vector pair. For all consecutive learning steps, we only searched in the neighbourhood of the old winner neuron for each source spectrum $\boldsymbol{x}(j)$. Since the map is getting more stable with every learning step (due to decreasing $\sigma(t)$ ) and changes are more subtle during the fine-tuning phase, we can lower the search radius $r_{\text {search }}(t)=(1-t) \sqrt{N} / 2+2$ with increasing number of learning steps. The number of operations is then $\sim(1-t) N / 4$ per learning step until we reach $\sim N$ operations in the last step.

The second technique reduces the number of adaption steps performed by Eq. (2) by defining a threshold. Now the neuron $\boldsymbol{m}_{i}$ is adapted only if the neighbourhood function exceeds a predefined value $\tilde{\alpha}$, i.e.

$\boldsymbol{m}_{i}(T+1)=\left\{\begin{array}{lll}\boldsymbol{m}_{i}(T) & \text { if } & h_{\mathrm{ci}} \leq \tilde{\alpha} \\ \boldsymbol{m}_{i}(T)+h_{\mathrm{ci}}(T)\left[\boldsymbol{x}-\boldsymbol{m}_{i}(T)\right] & \text { if } & h_{\mathrm{ci}}>\tilde{\alpha}\end{array}\right.$

where we used $\tilde{\alpha}=\alpha_{\text {end }} / 100$.

\subsubsection{Number of iteration steps and convergence behaviour}

We illustrate the convergence behaviour in two ways. First, Fig. 5 shows the average travel distance of all source spectra. Between each two subsequent learning steps we sum up all location vector changes of each source spectrum in the SOM. At certain learning steps, especially in the early training phase, major reorganisations within the map occur. Such points can be observed in the corresponding visualised maps (presented in the next section) at those particular steps.

Secondly, we calculate

$\chi^{2}(T)=\sum_{j=0}^{k}\left(\boldsymbol{x}(j)-\boldsymbol{m}_{j c}(T)\right)^{2}$

between the source spectra $x(j)$ and their corresponding best matching weight vectors $\boldsymbol{m}_{j c}$ for each learning step $T$. If 


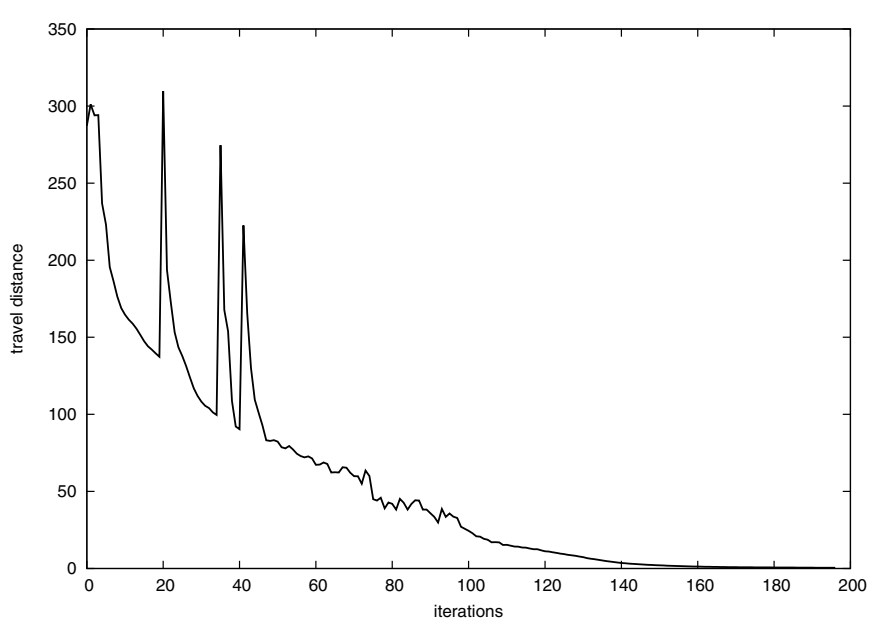

Fig. 5. Change in average travel distance (thus the change from one location vector on the map to another) of all source spectra.

$\chi^{2}$ ceases to drop, we can abort the learning process at this point. Then the network has reached its optimal point between plasticity and stability where the weight vectors still form a smooth landscape. We found that the map settles after 200 learning steps. Jumps of source spectra to different locations are rare in the last learning steps.

\section{Analysis methods}

\subsection{Map visualisation and blending in physical properties}

\subsubsection{Visualisation and presentation of the spectral database}

After the computation of the SOM finished we built a system that connects all the given information and present it in an userfriendly way. This system allows the user (1) to browse and navigate within the large spectral database; (2) to find relations between different objects; (3) to search for similar objects from a real or artificial template spectrum.

Each object is represented by an icon that shows its spectrum. The background colour encodes the flux density averaged over the spectrum, which can be used as a proxy for the signal-to-noise ratio in the spectrum ${ }^{6}$.

Each object is linked to a summary page that shows the top 20 most similar spectra. As similarity measure we use the simple Euclidean distance. And finally, each object is linked to the SDSS SkyServer Object Explorer ${ }^{7}$ where additional information can be retrieved. Figure 6 displays the blowup of $30 \times 30$ spectra from the icon map including a cluster of carbon stars located in the upper left. White areas show unoccupied cells without source spectra.

In addition to the icon map, other representations of the SOM are possible: (1) the difference between the network weights and the corresponding input spectra in a logarithmic scale;

\footnotetext{
6 There is a strong correlation between the signal-to-noise ratio and the fiber magnitudes. See http://www.sdss.org/dr6/products/ spectra/snmagplate.html. The average flux density in the spectrum, which corresponds to a fiber magnitude measured over the whole spectroscopic wavelength window, can thus be used as a proxy for the $\mathrm{S} / \mathrm{N}$

7 http://skyserver.sdss.org/public/en/tools/explore/ obj .asp
}

(2) the unified distance matrix (Sect. 4.1.2); and (3) the $z$ map (Sect. 4.1.3) using the redshifts from the SDSS spectro pipeline. We then calculated what we call a "difference map" for each spectrum. The difference map colour codes for each single spectrum in the SOM its measure of similarity to a given "template" spectrum $\boldsymbol{y}$ which can be either real or artificial as long as it matches the same spectral window and resolution. Such a map is calculated for every grid cell within the network with

$d(i)=\log (\|\boldsymbol{x}(i)-\boldsymbol{y}\|+1) / \log \left(\max _{j}\{\|\boldsymbol{x}(j)-\boldsymbol{y}\|\}+1\right)$,

where $\boldsymbol{x}(i)$ denotes the spectrum attached to position $i$ in the SOM and $d(i)$ is the difference value in the range $[0,1]$ that can be mapped to any colour gradient. For example, Fig. 7 shows the difference map for the M6 star SDSS J092644.26+592553.5, which is located in the lower left corner. Such difference maps provide a useful tool to identify objects that are located in different parts of the SOM, even though their spectral types are similar. Lighter regions in Fig. 7 show a high degree of dissimilarity, darker regions show a high degree of similarity. Grey areas mark free space in the map that is not occupied with spectra. The dark blue area in the lower left shows an identified cluster of late-type stars.

\subsubsection{Unified distance matrix}

The most common visualisation of this particular network is the unified distance matrix ( $U$ matrix) showing the distance between neighbouring neurons within the map (Ultsch \& Siemon 1990). The $U$ matrix is calculated for each weight vector $\boldsymbol{m}_{i}$ as the sum of distances of all four immediate neighbours normalised by the maximum occurring sum of these distances. The right panel of Fig. 7 shows the $U$ matrix of the network on a logarithmic scale at the final learning step. Lighter colours in the map indicate a high degree of variation, in contrast darker areas indicate similar weight vectors and clusters of similar objects. Bigger "mountains" (light colours), i.e. larger distances between neurons, indicate a large dissimilarity between clusters, smaller mountains indicate similar clusters.

When searching for unusual objects, very small clusters and areas of high variation can be of particular interest. The variation is highest at the cluster boundaries. Boundary regions are usually not occupied with source spectra because the neuronal landscape changes there from one type to another (see also Fig. 4). This map is only calculated from the artificial spectra but gives a good indication where a lot of change happens, a good indicator to find unusual objects.

\subsubsection{Mapping of physical properties}

In order to gain a deeper understanding of the SOM, we visualised several physical properties. In total, we could gather over thirty different maps that describe various relationships between different spectral types. Here we discuss three examples. First, a photometric object classification parameter is colour-coded. Then, we plot the spectroscopic object classification. Finally the distribution of the redshift over the SOM is analysed.

The SDSS consists of two surveys, the imaging survey in five specially designed photometric bands and the spectroscopic survey of objects selected from the catalogues that were derived from the high-quality five-colour photometry and the analysis of the image structure. The completely automated algorithm of the target selection results in a classification of objects as candidates 
A. in der Au et al.: ASPECT: A spectra clustering tool for explorationof large spectral surveys
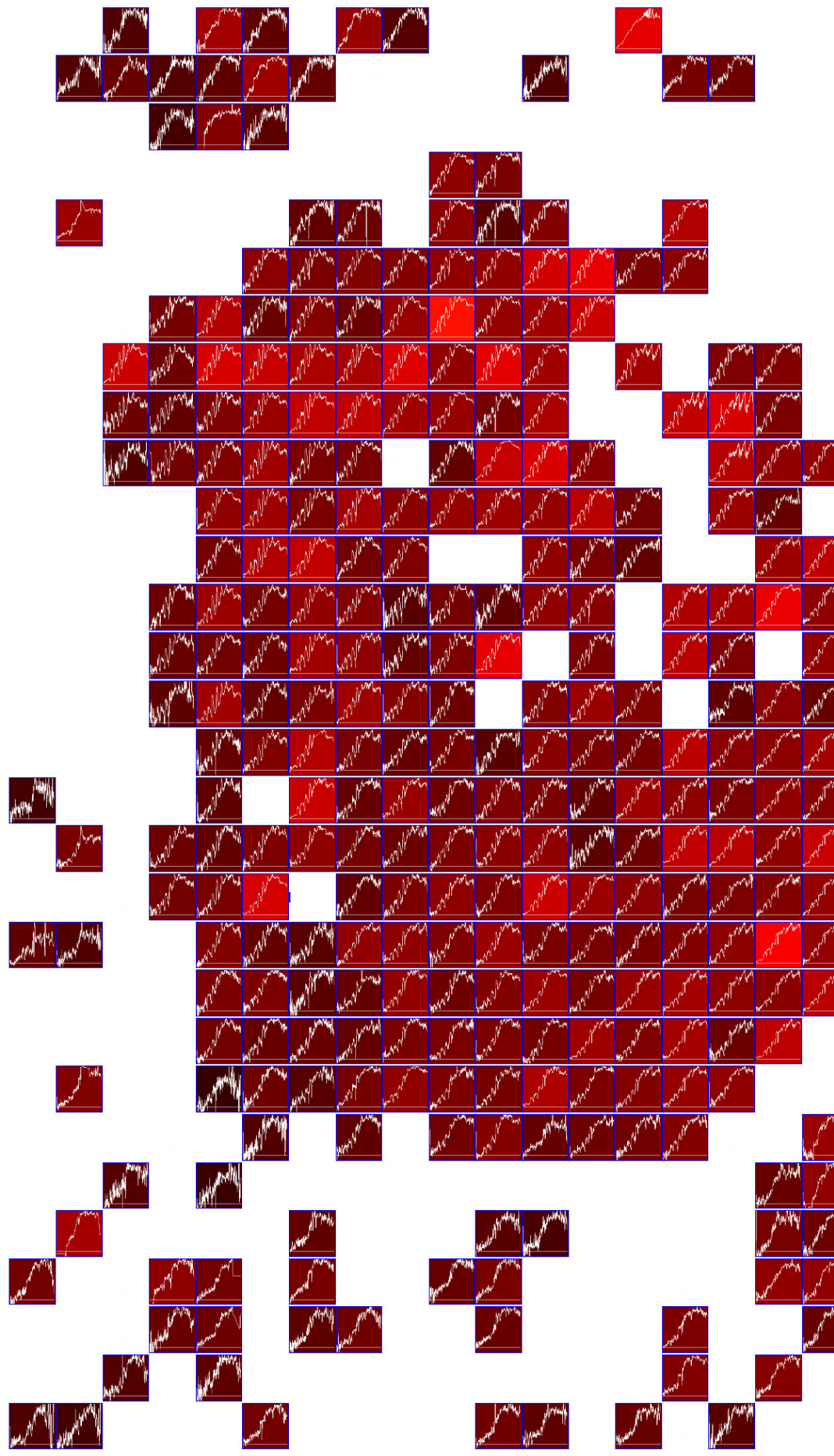
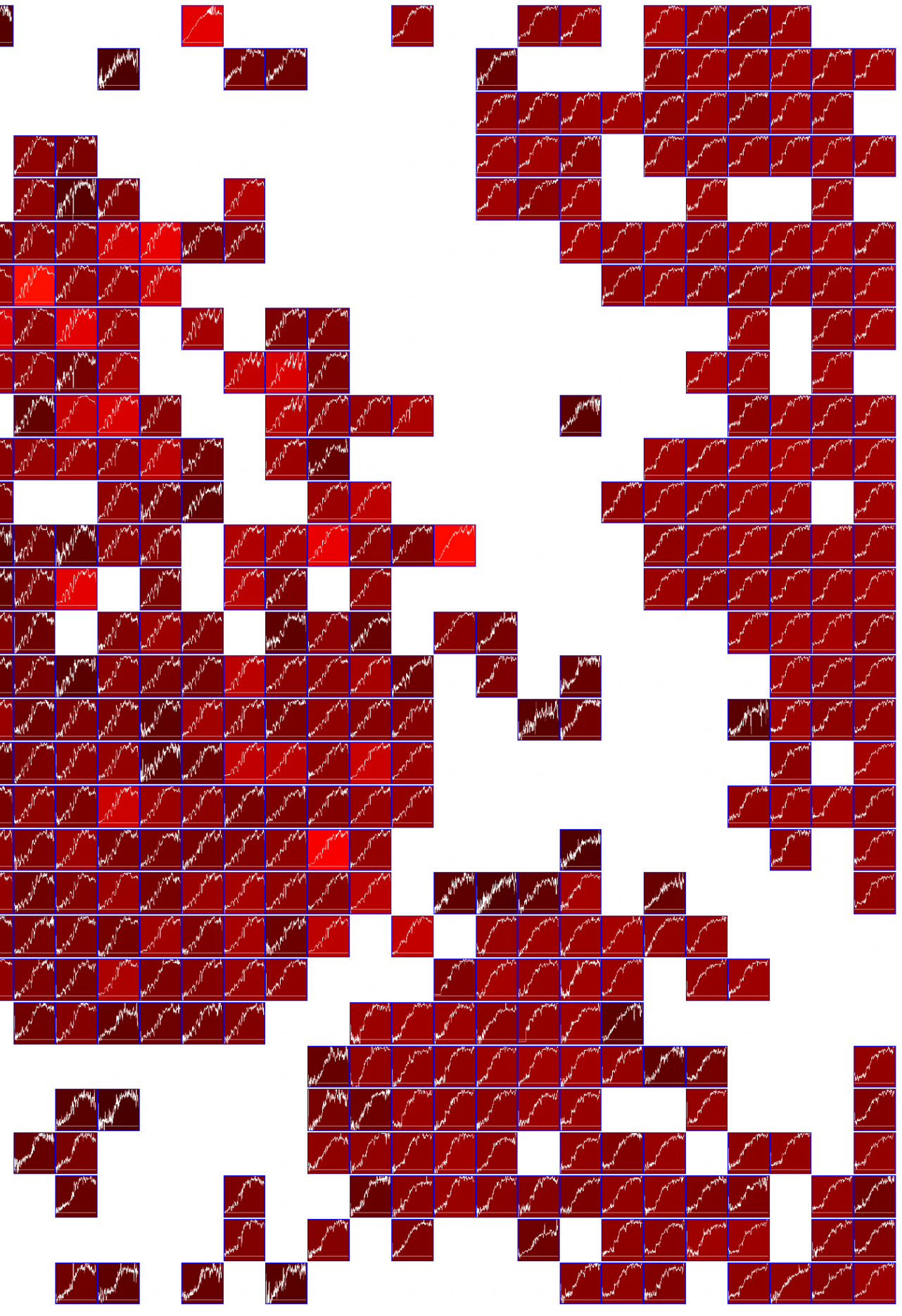

Fig. 6. Cutout from the icon map including a cluster of carbon stars.

for various types of galaxies, stars, or quasars. This information is coded in the target selection flag that is used by the SDSS for the selection of the spectroscopic targets. In other words, the target flag stores what that reason was for taking a spectrum. In general, the "primary" selection target bits denote science targets, and the "secondary" target bits denote spectrophotometric standards, sky targets, and other technical targets. Detailed descriptions of the overall target selection algorithm are given by Stoughton et al. (2002), Eisenstein et al. (2001), Richards et al. (2002), and Strauss et al. (2002).

The left panel of Fig. 8 displays the object classification based on the primary target selection flag. The colours are attributed to object types as described on the bottom of the panel. For clarity, several similar object types were combined (for example, the target flags QSO_CAP, QSO_SKIRT, QSO_FIRST_CAP, and QSO_FIRST_SKIRT were merged to the type QSO=quasar). HIZ QSO means high-z quasar, LRG means luminous red galaxy. Objects with multiple target flags are marked black. The most interesting property of this figure is the clear separation of the different object types. Within the larger clusters, we observe subtle but continuous changes in the shape of the continuum and the properties of the emission lines. Quasar candidates populate a fragmented area at the bottom, but also a number of isolated clumps scattered across the map. This is to be expected 

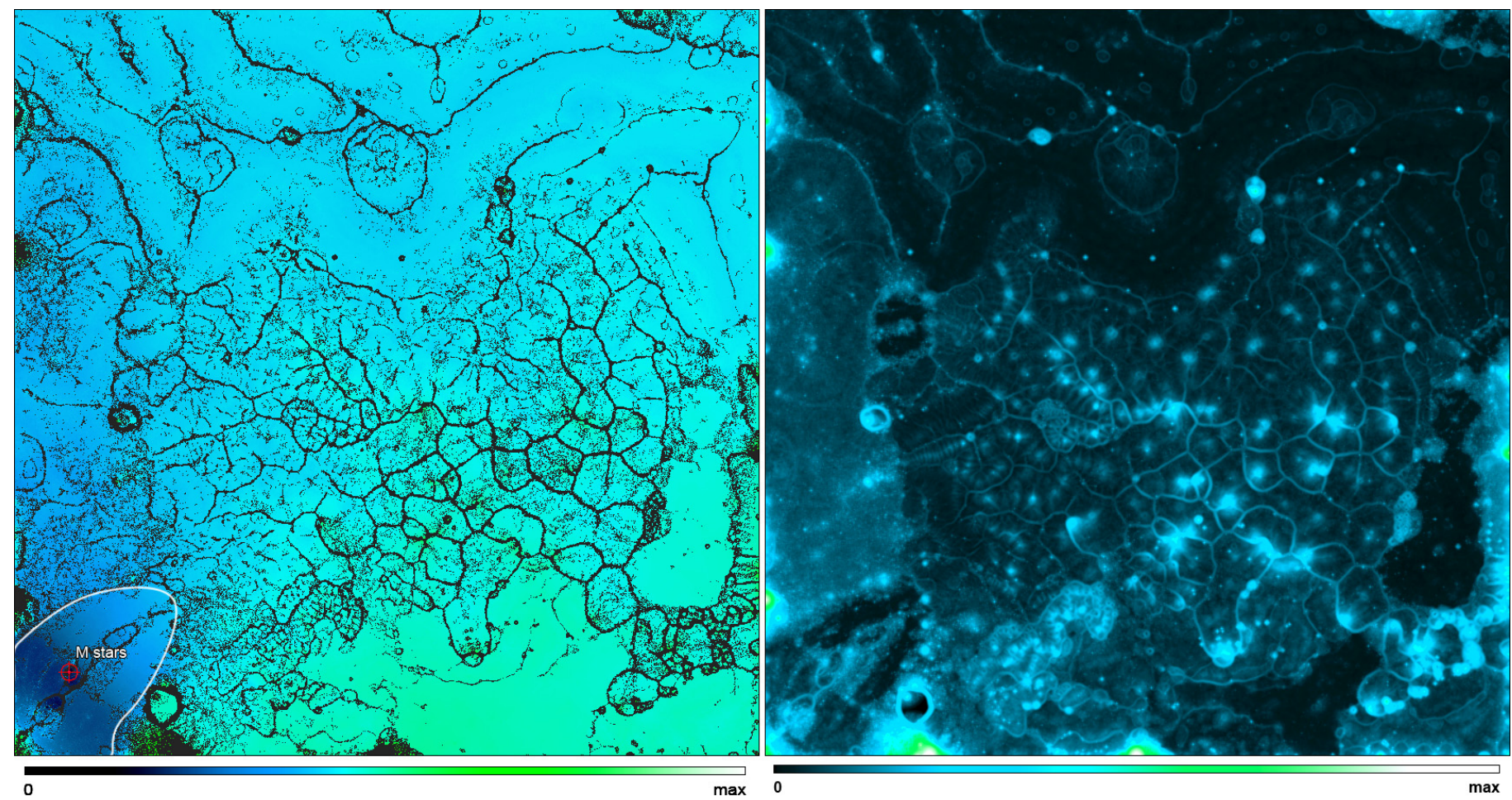

Fig. 7. SOM for $\sim 6 \times 10^{5}$ spectra from the SDSS DR4. Left: difference map for the M6 star SDSS J092644.26+592553.5. Right: U matrix of the SOM on logarithmic scale.
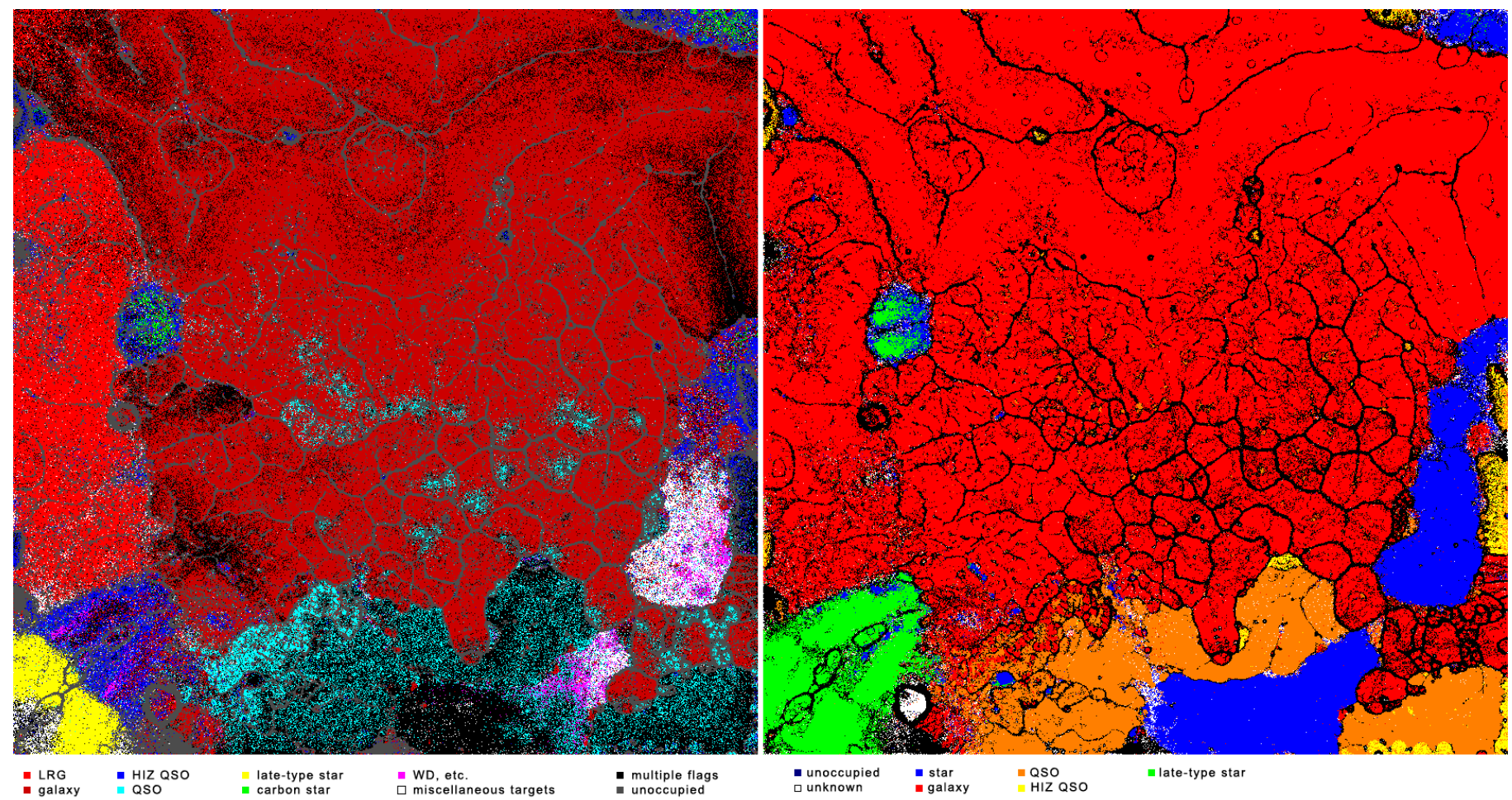

Fig. 8. The same SOM as in Fig. 7, but with colour coding representing the SDSS primary target selection flag (left) and the classification parameter specClass resulting from the spectroscopic pipeline (right).

as a consequence of the wide redshift range covered by the SDSS quasars (see below).

Typically, the parameter specClass should be used to characterise the object type. The class attribute was set by the spectroscopic pipeline of the SDSS after the spectrum was observed. The following classes are used: star, late-type star, galaxy, emission line galaxy, quasar (QSO), high-z quasar (HIZ QSO), and unknown (for unclassifiable spectra). Object type classification by the SDSS spectroscopic pipeline is discussed in Stoughton et al. (2002). The visualisation of the class attribute in the right panel of Fig. 8 underlines the separation of object types in our SOM even stronger than the left panel. An interesting 
A. in der Au et al.: ASPECT: A spectra clustering tool for explorationof large spectral surveys

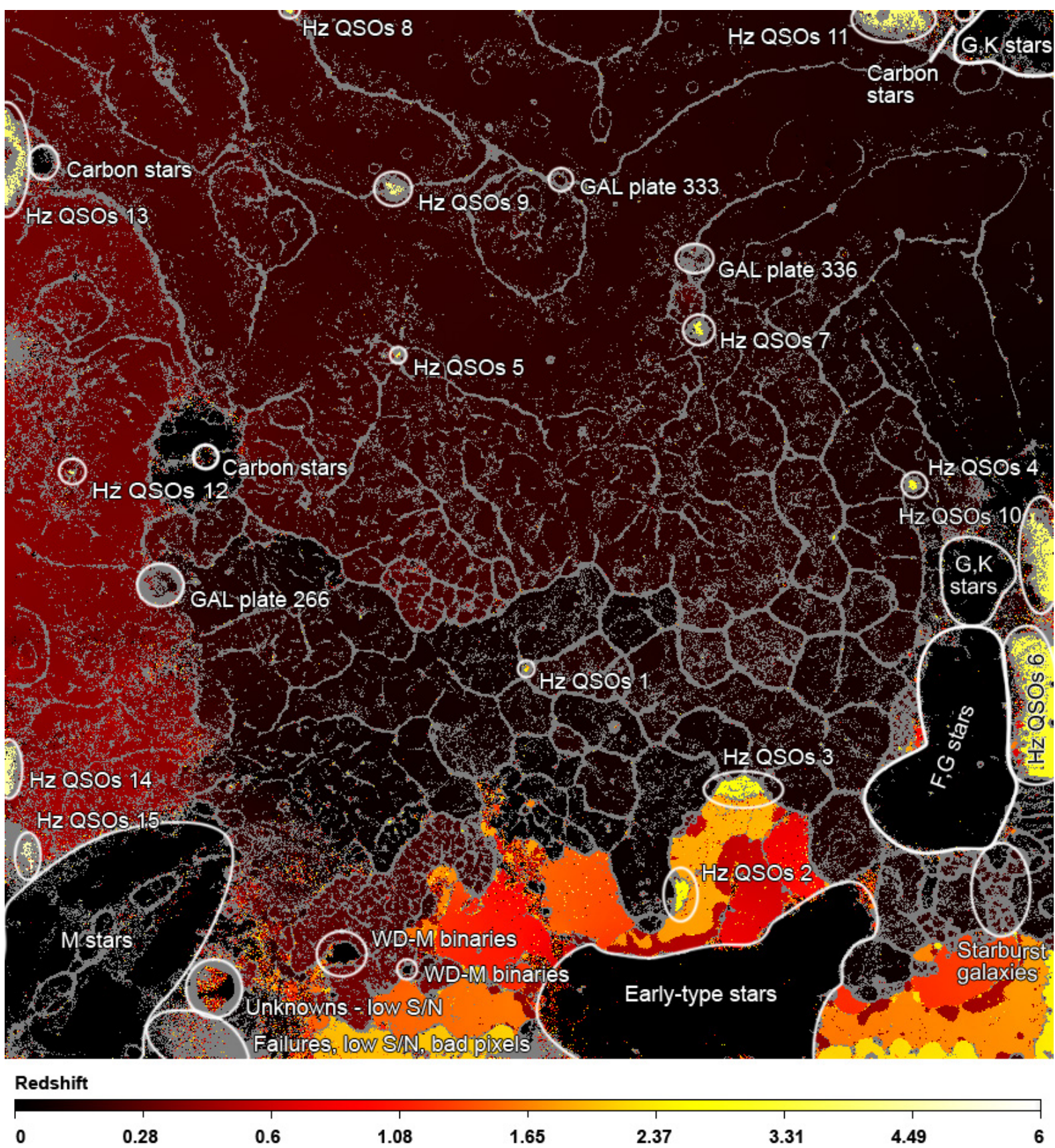

Fig. 9. The $z$ map with redshifts derived by the SDSS spectroscopic pipeline. Grey areas mark free space in the map that is not occupied with spectra. We labelled some regions that show high concentrations of particular objects types.

detail is the strong clustering of the unknown spectral types at the bottom left. The vast majority of these spectra suffer from a low signal-to-noise ratio. The lower left corner of the map is populated by late-type stars. The comparison with the left panel reveals that many of them were targeted as high- $z$ quasars. This is caused by the similarity of the broad-band colours of these two different object types (see below).

For an extragalactic survey like SDSS, one of the most interesting visualisations is the $z$ map that highlights the redshifts $z$ derived by the spectroscopic pipeline of the SDSS (Fig. 9). Since the spectra were not transformed into their rest-frames, a strong ordering and cluster formation towards redshifts can be observed for galaxies and quasars. We visually inspected a representative number of spectra from each of the most striking clusters in the
SOM to check out the spectral types. The result is illustrated by the labels in Fig. 9.

The SDSS quasars cover a redshift interval from $z \sim 0$ to $\sim 6$ and form several distinct clusters corresponding to different $z$ intervals. This clustering is a natural consequence of redshifting the strong emission lines and a demonstration of the colour- $z$. relation of quasars. Quasars with $z \lesssim 2$ populate spatially adjacent areas on the SOM but also show a clear separation of different $z$ intervals (see the colour bar at the bottom of Fig. 9). In addition, we identified 15 separate clusters of high- $z$ quasars which were labelled in Fig. 9 and listed in Table 2. A particularly strong spectral feature is the continuum drop-off shortward of the Lyman $\alpha$ line at $1216 \AA$ (Lyman break) that is caused by the efficient absorption of UV photons by hydrogen atoms along 
Table 2. High-redshift quasar clusters.

\begin{tabular}{lccccc}
\hline \hline No. & Quantity & $z_{\text {mean }}$ & $\sigma$ & $z_{\min }$ & $z_{\max }$ \\
\hline 1 & 18 & 2.01 & 0.8 & 0.0 & 2.62 \\
2 & 165 & 2.66 & 0.07 & 1.88 & 2.72 \\
3 & 343 & 2.8 & 0.05 & 2.75 & 2.88 \\
4 & 34 & 2.9 & 0.56 & 0.07 & 3.16 \\
5 & 9 & 3.05 & 0.77 & 0.86 & 3.38 \\
6 & 2117 & 3.13 & 0.14 & 2.81 & 3.45 \\
7 & 51 & 3.21 & 0.17 & 2.98 & 4.24 \\
8 & 13 & 3.51 & 0.03 & 2.93 & 3.62 \\
9 & 65 & 3.82 & 0.86 & 0.0 & 4.32 \\
10 & 634 & 3.61 & 0.18 & 0.16 & 3.93 \\
11 & 385 & 3.81 & 0.26 & 0.52 & 4.06 \\
12 & 8 & 3.94 & 0.04 & 3.88 & 4.0 \\
13 & 344 & 4.06 & 0.19 & 3.53 & 4.42 \\
14 & 226 & 4.46 & 0.07 & 2.33 & 4.75 \\
15 & 84 & 4.85 & 0.3 & 3.7 & 5.41 \\
\hline
\end{tabular}

the line of sight. The Lyman break enters the SDSS spectral window at $z \gtrsim 2.2$ and moves towards longer wavelengths with increasing $z$. For redshifts $z \gtrsim 4.5$, the continuum is suppressed by the Lyman $\alpha$ forest shortward of $\lambda \sim 6700 \AA$ and practically completely absorbed by Lyman limit absorption shortward of $\lambda \sim 5000 \AA$. At these redshifts, the optical broad-band colours of the quasars become similar to those of late-M stars. It is thus not surprising that the highest- $z$ quasars clump on the SOM in the immediate neighbourhood of the M stars.

However the SOM cannot preserve all possible topologies in its two dimensions because of the high dimensions of the input spectra. A map in three dimensions would allow better arrangements of clusters and more topology information would be preserved. On the other hand it would be more difficult to grasp and visualise and may require specialised visualisation software. Speckmann et al. (1994) investigated the dimensionality of input datasets and its effect on topology preservation of the SOM.

\subsection{Tracking of catalogues}

For the vast majority of stars, galaxies, and quasars, the spectral properties vary smoothly over the SOM because stellar spectral types, stellar populations, redshifts, and dust reddening are continuously distributed in the spectroscopic database of the SDSS. The bulk of the spectra thus forms large coherent areas interspersed with small areas of "no man's land" occupied either by a mixture of various object types or by more or less rare types with pronounced spectral peculiarities (as well as by spectra of low $\mathrm{S} / \mathrm{N}$ or strongly disturbed spectra). If these peculiarities are made of characteristic broad features at fixed wavelengths in the observer frame, the spectra tend to form small clusters. Though it is not easy to specify the relationship between the clustering behaviour and the spectral properties, the very fact of such a clustering is useful for efficiently searching such rare objects once a cluster has been identified, e.g. by an input catalogue of known objects of that type.

\subsubsection{Carbon stars}

First, we choose the relatively rare type of carbon stars which display prominent (Swan) bands of $\mathrm{C}_{2}$ in their spectra. We use two "input catalogues" to trace such objects in the SOM: the catalogue of 65 DQ white dwarfs from Koester \& Knist (2006) and the catalogue of faint high-latitude carbon (FHLC) stars from Downes et al. (2004). The latter catalogue lists $251 \mathrm{C}$ stars of which 231 are in our database. We are interested how the objects from either catalogue are located relative to each other on the SOM.

A clump of catalogue objects is defined to form a cluster if each member is located at a distance $\leq 15$ cells from another cluster member. The distribution over the SOM for the objects from the two catalogues is shown in Fig. 10 where the four richest clusters are labeled. The percentage of objects concentrated in the four largest clusters are given in Table 3. Objects that do not fall in one of these clusters are listed as scattered.

\section{- DQ white dwarfs (Koester $\mathcal{E}$ Knist 2006):}

White dwarfs of spectral type DQ are defined as showing absorption features of carbon atoms or molecules which are believed to be dredged-up from the underlying carbon/oxygen core to the surface by a deepening helium convection zone. Among others, DQs are of special interest because they provide information about the deeper layers of white dwarfs.

The DQ stars are clustered at the borders of the area populated by quasars with redshifts around 1 . This can be understood primarily as due to their blue continua. Moreover, the $\mathrm{C}_{2}$ Swan bands resemble broad absorption lines in quasar spectra (e.g., SDSS J020534.13+215559.7; Meusinger et al. 2012), and even broad quasar emission lines can be mimicked by the absorption troughs in the case of very strong bands. Though not very compact, the three richest DQ clusters contain $80 \%$ of the catalogue objects.

We used the objects from the input catalogue as tracers to search for similar spectra in their neighbourhood. Since the SOM areas populated by the input catalogue objects do not show welldefined boundaries, we defined a local neighbourhood around each single catalogue object by the 8 next neighbours. This yields a list of 365 objects. From the quick evaluation of the individual spectra we found the following composition of this quite inhomogeneous mixture of object types: (1) 153 mostly (93\%) catalogued white dwarfs and 14 catalogued subdwarfs; (2) 105 extragalactic objects (95 quasars, 4 BL Lac objects, 6 galaxies); and (3) 93 unclassified, not catalogued objects, mostly (84\%) with featureless blue spectra (probably DC white dwarfs). The first group includes $22 \mathrm{DQs}$ from the input catalogue, 19 objects were found to be classified as DQ by Eisenstein et al. (2006), another 3 objects are probably new DQs, yet with only weak und thus uncertain carbon features. 116 objects from group 1 are catalogued white dwarfs of other types, mostly DC or DA.

In Fig. 11, we compare the median input spectrum with the median spectrum of the DQ white dwarfs which were "discovered" by this method. This exercise shows that, even for weakly clustering objects of a rare type, new members can be discovered efficiently by checking the local SOM neighbourhood of known objects.

\section{- Faint high-latitude carbon stars Downes et al. (2004):}

FHLCs were considered interesting, among others, as they are believed to be tracers of the Galactic halo, though recent studies have shown that only a fraction of them are distant halo giants whereas another significant fraction, maybe the majority, are nearby dwarfs. The empirical database of the FHLCs has grown substantially with the SDSS.

Compared to the DQs, the FHLC stars from Downes et al. (2004) populate completely different areas of the SOM in the neighbourhood of intermediate and late-type stars or high- $z$ quasars, respectively. $66 \%$ of the catalogue objects are found 

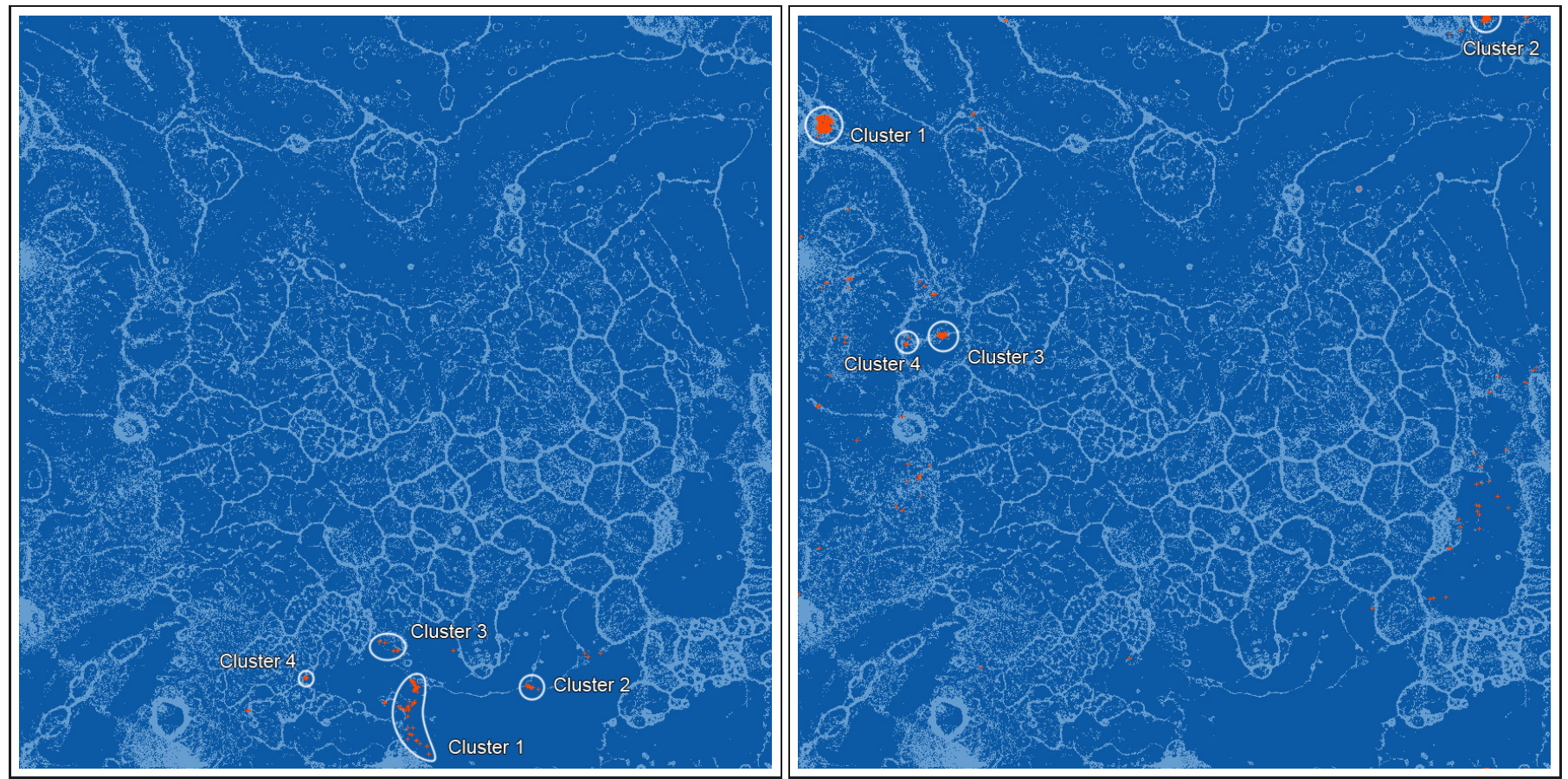

Fig. 10. SOM object positions and clusters for the white dwarfs of spectral type DQ from Koester \& Knist (2006) (left) and the faint high-latitude carbon stars from Downes et al. (2004) (right).

Table 3. Clustering behaviour of catalogued carbon stars.

\begin{tabular}{ccc}
\hline \hline & DQ & FHLC \\
& $(1)$ & $(2)$ \\
\hline Total number of objects & 65 & 231 \\
Percentage of objects in cluster 1 & 58.5 & 45.5 \\
$\ldots$.. in cluster 2 & 12.3 & 12.5 \\
$\ldots$.. in cluster 3 & 9.2 & 9.1 \\
... in cluster 4 & 6.1 & 2.2 \\
$\ldots$... scattered & 13.9 & 30.7 \\
\hline
\end{tabular}

References. (1) Koester \& Knist (2006); (2) Downes et al. (2004).

to be concentrated in three distinct clusters with well-defined boundaries. There are subtle differences between the mean spectra of the three clumps. $\mathrm{C} 2-\mathrm{C} 3-\mathrm{C} 1$ form a kind of a spectral sequence where $\mathrm{C} 3$ is of later type than $\mathrm{C} 2$ and $\mathrm{C} 1$ is of later type than C3. The clusters do not include the stars with the weakest absorption bands, but most of the stars with very pronounced $\mathrm{C}_{2}$ bands are included, though some of them are scattered across the map.

\subsubsection{High-Redshift Quasars}

As discussed already in Sect. 4.1.3, high-z quasars strongly tend to clump on the SOM. Here we consider the highest- $z$ quasar cluster 15 (Fig. 9, Table 2) for illustration. This well-defined cluster consists of 84 objects, among them are 78 quasars with $z>4$.7. For the same redshift range, the SDSS DR7 quasar catalogue (Schneider et al. 2010) contains 125 quasars with plate numbers $\leq 1822$, which is the highest plate number in the DR4 database used for our SOM. The completeness of the cluster is thus $62 \%$, which is somewhat better than for the biggest clusters of DQWDs and FHLCs, respectively (Table 3). The fact that more than one third of the highest- $z$ quasars are scattered across the SOM is not surprising since their spectra can be quite different (Fig. 12).

From the individual inspection of the spectra of all $84 \mathrm{ob}-$ jects we found that 82 spectra are in fact quasars with $z \sim 4$ to 5. Another object, SDSS J153708.14+315854.0, is likely a galaxy at $z \sim 0.612$, but the $\mathrm{S} / \mathrm{N}$ in the spectrum is low and so is the redshift confidence $(\mathrm{zConf}=0.69)^{8}$. For another object, SDSS J084348.13+341255.4, the red part of the spectrum is so much disturbed that a classification is impossible. Hence, the search for highest- $z$ quasars in cluster 15 yields a success rate as high as $98.8 \%$.

\section{Other applications}

\subsection{Quasars}

The advent of large spectroscopic surveys has resulted in an increase of the number of catalogued quasars by more than one order of magnitude. The Fifth Edition of the SDSS Quasar Catalogue (Schneider et al. 2010) contains 105783 entries. For the vast majority, the individual spectra largely agree with the quasar composite spectrum produced by averaging over large quasar samples, i.e. a blue UV/optical continuum and strong broad emission lines. However, these surveys revealed also examples of quasars showing dramatically different spectral properties never seen before, such as very complex systems of absorption features in FeLoBAL quasars (Hall et al. 2002), very weak or undetectable UV emission lines (Shemmer et al. 2009), extremely red continua (Glikman et al. 2007; Urrutia et al. 2009),

\footnotetext{
8 The contamination of the high- $z$ quasar cluster with a galaxy of such a low redshift is not unexpected because the $4000 \AA$ break of the galaxy spectrum can be easily confused with the Lyman break when the spectrum is noisy.
} 

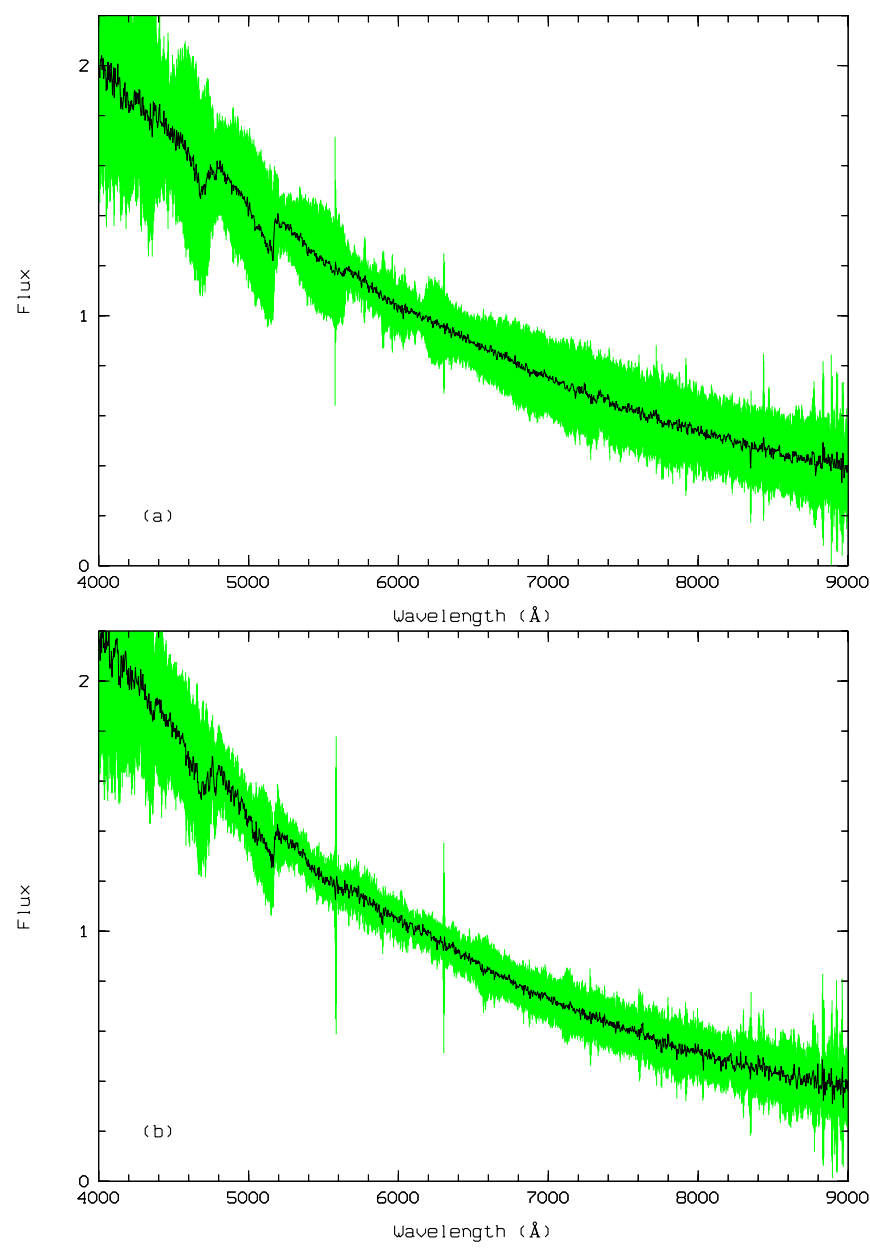

Fig. 11. Median and standard deviation for the spectra of DQ white dwarfs found in the 8-cell neighbourhood of objects from the catalogue of Koester \& Knist (2006): a) for 22 objects which are in the input catalogue; and b) for another 22 similar spectra which are not.

or "mysterious" objects with spectra that are difficult to explain (Hall et al. 2002). Such rare types may be related to special evolutionary stages of the quasar phenomenon and are expected to shed light on the evolution of active galactic nuclei and their feedback on the evolution of the host galaxies.

We started a systematic search for such outliers in the data archive of about $10^{5}$ spectra classified as quasars with $z=$ 0.6 to 4.3 by the spectroscopic pipeline of the SDSS DR7 (Meusinger et al. 2012). The SOM technique provides us with a unique opportunity for efficiently selecting rare spectral types from this huge data base. The SOM of the complete sample is expected to separate the quasars according to their redshifts (see Fig. 9). As it was our aim to separate the unusual spectra, we applied the SOM method to subsamples binned into $z$ intervals. A bin size of $\Delta z=0.1$ was chosen to ensure that the differences between the spectra, as seen by the SOM, caused by their different redshifts are smaller than the differences due to the spectral peculiarities. The size of the SOMs strongly varies with $z$ between 196 and 8281 neurons. As outliers tend to settle at the edges and corners of the maps, they were easily identified by means of the visual inspection of the icon maps of the 37 SOMs. We selected 1530 objects which were individually analysed to reject contaminants (rare stellar spectral types, spectra with too low $\mathrm{S} / \mathrm{N}$, quasars with wrong $z$ from the SDSS pipeline), to reestimate the redshift, and to characterise the peculiarities of the spectra.
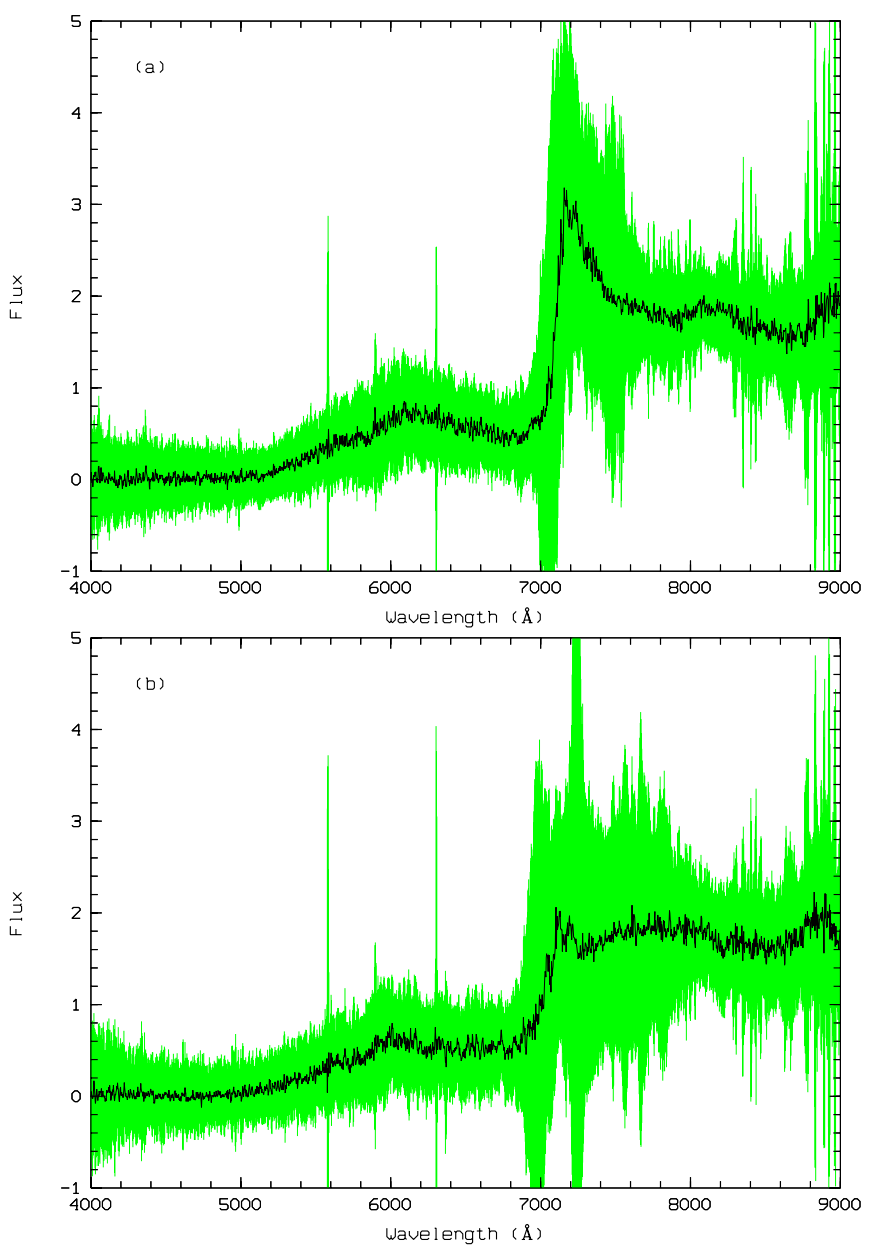

Fig. 12. Median and standard deviation for the spectra of the high redshift quasars with $z>4.7$ in cluster 15 a) and outside of cluster 15; b) respectively.

The final catalogue contains 1005 unusual quasars, which could be classified into 6 different types plus a small group of miscellaneous objects ${ }^{9}$. Though our catalogue is not complete in a quantifiable sense, it provides the largest compilation of unusual quasar spectra so far. In particular, the results support the idea that these peculiar quasar spectra are not just "oddballs", but represent quasar populations which are probably underrepresented in the presently available quasar samples.

\subsection{Galaxy Zoo: visualisation of external catalogues}

Galaxies account for about three quarters of the SDSS spectra. For the understanding of galaxies, structure information is crucial and is, in contrast to quasars and stars, in principle available from the SDSS images. Galaxy morphology is usually encoded by the morphological type which is a powerful indicator for the spatial distribution of stars and therewith for the dynamical evolution of the system, including its merger history. To gain further insight into the distribution of the galaxies in the SOM, it may thus be useful to overplot the morphological type information.

Simple morphological classifications were collected by the Galaxy Zoo project (Lintott et al. 2011a) for 893212 objects of SDSS Data Release 6. This huge project was possible thanks

9 The spectral atlas for these quasars is available at http://www.tls-tautenburg.de/fileadmin/forschung/meus/ AGN/Unusual_quasars.html 
A. in der Au et al.: ASPECT: A spectra clustering tool for explorationof large spectral surveys
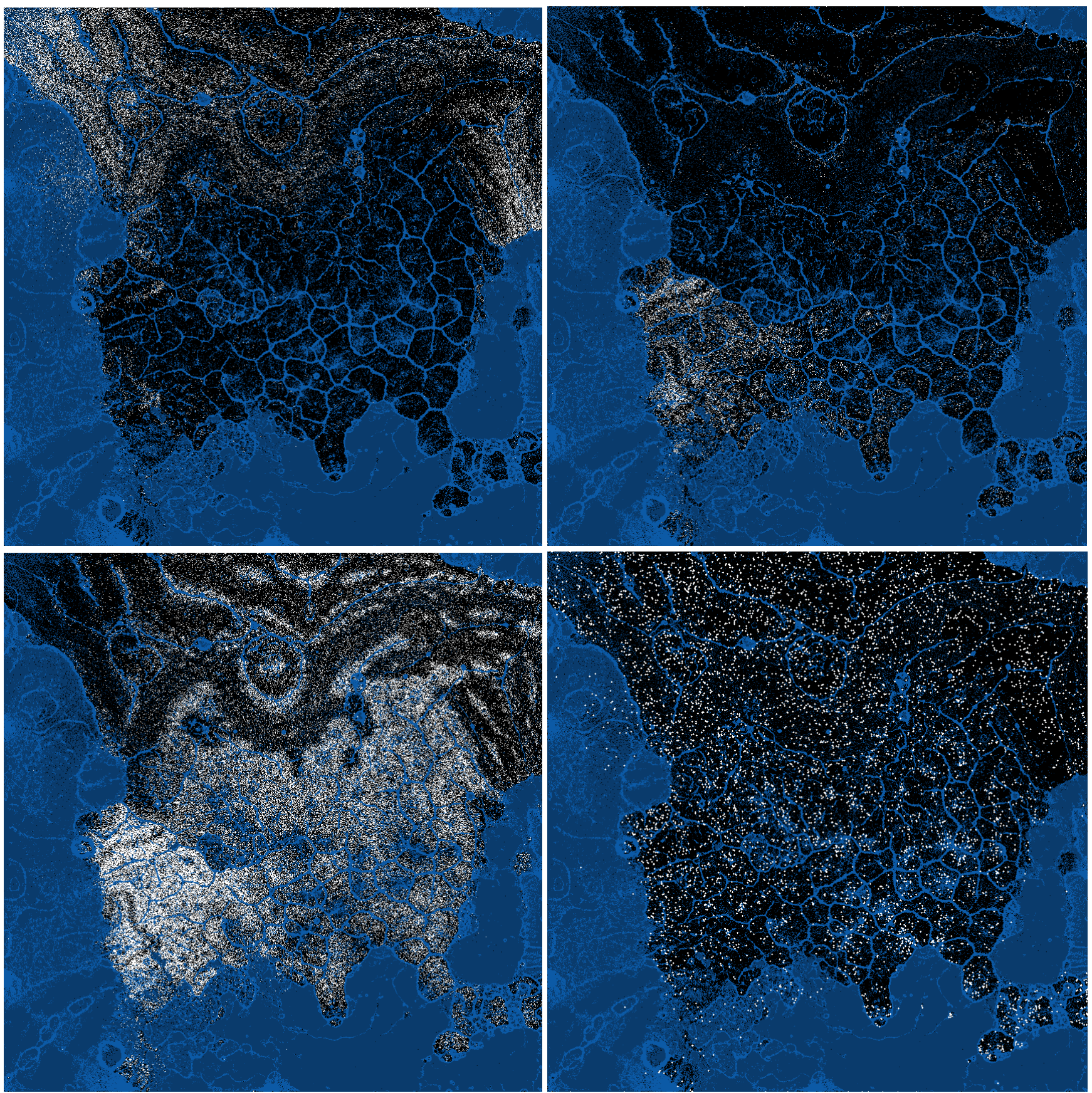

Fig. 13. The same SOM as in the previous figures where the morphological type flags from the Galaxy Zoo project (Lintott et al. 2011a) are highlighted as white dots. Left to right then top to bottom: elliptical galaxies, edge-on spirals, spirals (clockwise, anti-clockwise, edge-on), merger.

to the involvement of hundreds of thousands volunteer "citizen scientists". The galaxies were inspected on composite gri band SDSS images to derive one of the six classification categories: (1) elliptical galaxy; (2) clockwise spiral galaxy; (3) anticlockwise spiral galaxy; (4) other spiral galaxies (e.g. edge on); (5) star or don't know (e.g. artefact); (6) merger. The results were bias-corrected since faint or/and distant spiral galaxies are likely misclassified as ellipticals when the spiral arms are not or barely visible (Bamford et al. 2009).

We use the spectroscopically observed subsample of the Galaxy Zoo data. This results in 667945 objects in total and a subsample of 367306 objects that overlaps with the DR4 sample used for our SOM. The catalogue "Morphological types from
Galaxy Zoo 1" (Lintott et al. 2011b) lists the fraction of votes for the six classification categories. To turn those vote fractions into corresponding flags for elliptical or spiral galaxies requires $80 \%$ of the votes in that category; all other galaxies are classified as uncertain. For the classification as a merger, a lower threshold of 0.4 is sufficient (see Lintott et al. 2011a).

Figure 13 shows the distribution of the flags for (left to right then top to bottom) elliptical galaxies (E), edge-on spirals, spirals (S), and mergers. The redshift increases from right to left on large scales, but there are deviations on smaller scales. No flags are available for the objects at the middle of the left edge of the SOM between the $\mathrm{M}$ star region and the high- $z$ quasar clusters 14 and 15 at the bottom and the high- $z$ quasar cluster 13 at the top 


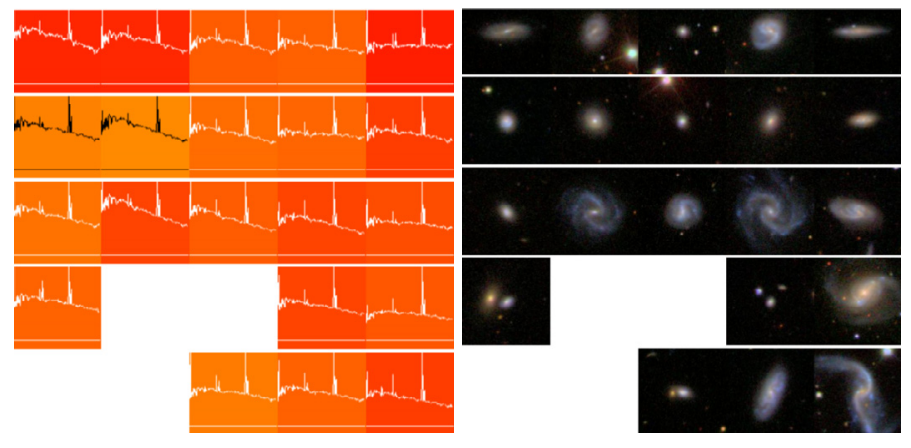

Fig. 14. Image cutouts from an icon map (left) and the corresponding picture map (right) of low-redshift galaxies.

(see Fig. 9). This region is occupied by the highest- $z$ galaxies where morphological information from the SDSS imaging is not reliable for the vast majority of the galaxies. Nearly all flagged galaxies within that area were assigned to type E.

A few interesting details can be recognised by the simple inspection of Fig. 13. First, E galaxies populate mostly the upper part of the SOM, the type $S$ is concentrated towards the lower half. However, there are no clear boundaries between the areas populated by $\mathrm{E}$ and $\mathrm{S}$ galaxies, respectively. In particular, the region in the middle of the upper part (around 12 o'clock) is populated by comparable fractions of $\mathrm{E}$ and $\mathrm{S}$ galaxies. On smaller scales, however, the two types are stronger separated in a kind of a meshwork structure. The cracks running through the high-density $\mathrm{S}$ area between about 7 and 9 o'clock are loosely populated by spectra of E-type galaxies and surrounded by a remarkable concentration of edge-on spirals. Finally, the spectra of merger galaxies do obviously not show a preference for any morphological type. Also, there is no enhanced population density of mergers in the clump of starburst galaxies labelled in Fig. 13. A few loose clumps of mergers are found at the boundary between galaxies and intermediate-redshift quasars, representing wet mergers with elevated star formation producing blue continua and strong emission lines. The detailed investigation of these issues is clearly beyond the scope of the present paper.

\subsection{Galaxy maps from SDSS DR7}

We finally note in passing that we computed SOMs for $\sim 8 \times$ $10^{5}$ galaxies from the SDSS DR7. As for the quasars in the previous subsection, the galaxies were binned into $z$ intervals with about 5000 spectra per bin. The analysis of the results is still in preparation. An additional powerful tool for the work with the resulting SOMs are picture maps (Fig. 14), i.e. representations of the SOMs where the colour images from the SDSS are displayed at the positions of the corresponding spectra. The comparison of the icon maps with the picture maps are expected to be helpful when searching for correlations between spectral properties and morphology or environment.

\section{Conclusions and future work}

In this paper we have presented ASPECT, a software tool that is able to cluster large quantities of spectra with the help of self-organising maps (SOMs; Kohonen 1982, 2001). We have built a topological map of 608793 spectra from the SDSS DR4 database to illustrate the capability of that tool. To explore the resulting topology information we have created a system that links each spectrum in the map to the SDSS DR7 explorer. ASPECT allows the user to browse and navigate through the entire spectral data set. Similarities within the SOM have been visualised with the help of the unified distance matrix (Ultsch \& Siemon 1990). Further we have introduced difference maps that colour code the similarity of a given template spectrum to all other spectra in the SOM. Data from different sources were mapped onto the resulting SOM. Especially the mapping of SDSS photometric and spectroscopic object types (Fig. 8) and SDSS derived redshifts (Fig. 9) onto the resulting SOM enable a better navigation within the data set.

Clusters of rare objects within the SOM can be identified either by the visual inspection of selected spectra or with the help of a given input catalogue of known objects of that type. The first method has been successfully applied for selecting unusual quasars from $10^{5}$ SDSS DR7 spectra in our previous study (Meusinger et al. 2012). Here we demonstrate the second method by means of 65 DQ white dwarfs from Koester $\&$ Knist (2006) and 231 Faint high-latitude carbon stars from Downes et al. (2004). From those catalogue objects $86 \%$ DQ white dwarfs and $69 \%$ FHLCs are concentrated in four major clusters respectively. By checking the SOM neighbourhood of those clusters similar objects can be discovered efficiently, even for weakly clustering objects. As another application we have mapped morphological information (i.e. galaxy types, mergers) from the Galaxy Zoo project onto the spectroscopic galaxy subsample of the SOM. As shown in Fig. 13, elliptical galaxies, spirals, and edge-on spirals show different distributions across the map. Merger galaxies, on the other hand, do not show a preference for any morphological type. More detailed galaxy morphology information, for example from the Galaxy Zoo2 project which data release is currently prepared but not yet available, is expected to offer interesting results when mapped to the here presented topology.

Data mining of other existing or upcoming massive spectroscopic surveys for instance the Sloan Extension for Galactic Understanding and Exploration (SEGUE; Yanny et al. 2009) or Apache Point Observatory Galactic Evolution Experiment (APOGEE) would offer great potential.

Further challenges involve the overcome of algorithmic limitations (runtime and memory bandwidth usage) of the current algorithm used in ASPECT. A distribution of the workload on modern supercomputers would enable the processing of even larger data sets. The source code is available on request for the interested reader.

Acknowledgements. We thank our anonymous referee and Dr. Polina Kondratieva for their important comments and suggestions. This research would be impossible without the use of data products from Sloan Digital Sky Survey (SDSS). Funding for the Sloan Digital Sky Survey (SDSS) has been provided by the Alfred P. Sloan Foundation, the Participating Institutions, the National Aeronautics and Space Administration, the National Science Foundation, the US Department of Energy, the Japanese Monbukagakusho, and the Max Planck Society. The SDSS Web site is http://www.sdss.org/. The SDSS is managed by the Astrophysical Research Consortium (ARC) for the Participating Institutions. The Participating Institutions are The University of Chicago, Fermilab, the Institute for Advanced Study, the Japan Participation Group, The Johns Hopkins University, the Korean Scientist Group, Los Alamos National Laboratory, the Max-Planck-Institute for Astronomy (MPIA), the Max-PlanckInstitute for Astrophysics (MPA), New Mexico State University, University of Pittsburgh, University of Portsmouth, Princeton University, the United States Naval Observatory, and the University of Washington. This research has made use of the SIMBAD database, operated at CDS, Strasbourg, France and of NASA's Astrophysics Data System Bibliographic Services. 
A. in der Au et al.: ASPECT: A spectra clustering tool for explorationof large spectral surveys

\section{References}

Abazajian, K. N., Adelman-McCarthy, J. K., Agüeros, M. A., et al. 2009, ApJS, 182,543

Adelman-McCarthy, J. K., Agüeros, M. A., Allam, S. S., et al. 2006, ApJS, 162, 38

Adelman-McCarthy, J. K., \& the SDSS Collaboration 2008, ApJS, 175, 297

Aihara, H., Allende Prieto, C., An, D., et al. 2011, ApJS, 193, 29

Andreon, S., Gargiulo, G., Longo, G., et al. 2000, MNRAS, 319, 700

Balastegui, A., Ruiz-Lapuente, P., \& Canal, R. 2001, MNRAS, 328, 283

Bamford, S. P., Nichol, R. C., Baldry, I. K., et al. 2009, MNRAS, 393, 1324

Bell, E., Davis, M., Dey, A., et al. 2009, in astro2010: The Astronomy and

Astrophysics Decadal Survey (Science White Papers), Astronomy No. 106

Berriman, G. B., \& Groom, S. L. 2011, Queue, 9, 21:20

Brett, D. R., West, R. G., \& Wheatley, P. J. 2004, MNRAS, 353, 369

Ciaramella, A., Donalek, E., Staiano, A., et al. 2005, Recent Research

Developments in Astrophysics (Research Signpost), 2, 27

Cottrell, M., \& Fort, J. C. 1987, Annales de l'Institut Henri Poincaré, 23, 1

Cottrell, M., Fort, J. C., \& Pagès, G. 1994, in ESANN, Brussels, Belgium, 235

Downes, R. A., Margon, B., Anderson, S. F., et al. 2004, AJ, 127, 2838

Eisenstein, D. J., Annis, J., Gunn, J. E., et al. 2001, AJ, 122, 2267

Eisenstein, D. J., Liebert, J., Harris, H. C., et al. 2006, ApJS, 167, 40

Erwin, E., Obermayer, K., \& Schulten, K. 1992, Biological Cybernetics, 67, 35

Geach, J. E. 2012, MNRAS, 419, 2633

Glikman, E., Helfand, D. J., White, R. L., et al. 2007, ApJ, 667, 673

Gray, J., Szalay, A. S., Thakar, A. R., et al. 2002 [arXiv:cs/0202014]

Hall, P. B., Anderson, S. F., Strauss, M. A., et al. 2002, ApJS, 141, 267

Hernandez-Pajares, M., \& Floris, J. 1994, MNRAS, 268, 444

Koester, D., \& Knist, S. 2006, A\&A, 454, 951

Kohonen, T. 1982, Biological Cybernetics, 43, 59

Kohonen, T. 2001, Self-Organizing Maps, 3rd edn. (New York: Springer)

Lesteven, S., Poinçot, P., \& Murtagh, F. 1996, Vistas Astron., 40, 395

Lintott, C., Schawinski, K., Bamford, S., et al. 2011a, MNRAS, 410, 166
Lintott, C., Schawinski, K., Bamford, S., et al. 2011b, VizieR Online Data Catalogue J/MNRAS/419/166

LSST Science Collaborations, et al. 2009 [arXiv:0912.0201]

Lupton, R., Gunn, J. E., Ivezić, Z., et al. 2001, in Astronomical Data Analysis Software and Systems X, eds. F. R. Harnden Jr., F. A. Primini, \& H. E. Payne, ASP Conf. Ser., 238, 269

Mähönen, P. H., \& Hakala, P. J. 1995, ApJ, 452, L77

Meusinger, H., Schalldach, P., Scholz, R.-D., et al. 2012, A\&A, 541, A77

Miller, A. S., \& Coe, M. J. 1996, MNRAS, 279, 293

Morales, I., Montero-Dorta, A. D., Azzaro, M., et al. 2012, MNRAS, 419, 1187

Naim, A., Ratnatunga, K. U., \& Griffiths, R. E. 1997, ApJS, 111, 357

Odewahn, S. C., Stockwell, E. B., Pennington, R. L., et al. 1992, AJ, 103, 318

Peacock, J. A., Schneider, P., Efstathiou, G., et al. 2006, ESA-ESO Working Group on Fundamental Cosmology, Tech. Rep.

Rajaniemi, H. J., \& Mähönen, P. 2002, ApJ, 566, 202

Richards, G. T., Fan, X., Newberg, H. J., et al. 2002, AJ, 123, 2945

Scaringi, S., Cottis, C. E., Knigge, C., \& Goad, M. R. 2009, MNRAS, 399, 2231

Schneider, D. P., Richards, G. T., Hall, P. B., et al. 2010, AJ, 139, 2360

Shemmer, O., Brandt, W. N., Anderson, S. F., et al. 2009, ApJ, 696, 580

Speckmann, H., Raddatz, G., \& Rosenstiel, W. 1994, in ESANN, Brussels, Belgium, 251

Stoughton, C., Lupton, R. H., Bernardi, M., et al. 2002, AJ, 123, 485

Strauss, M. A., Weinberg, D. H., Lupton, R. H., et al. 2002, AJ, 124, 1810

Tagliaferri, R., Longo, G., Milano, L., et al. 2003, Neural Netw., 16, 297

Torniainen, I., Tornikoski, M., Turunen, M., et al. 2008, A\&A, 482, 483

Ultsch, A., \& Siemon, H. P. 1990, in Proc. INNC'90, Int. Neural Network Conf. (Dordrecht, Netherlands: Kluwer), 305

Urrutia, T., Becker, R. H., White, R. L., et al. 2009, ApJ, 698, 1095

Way, M. J., \& Klose, C. D. 2012, PASP, 124, 274

Xue, J.-Q., Li, Q.-B., \& Zhao, Y.-H. 2001, Chinese Astron. Astrophys., 25, 120

Yanny, B., Rockosi, C., Newberg, H. J., et al. 2009, AJ, 137, 4377

York, D. G., Adelman, J., Johnn, E., Anderson, J., et al. 2000, AJ, 120, 1579 